\title{
Hypersensitivity pneumonitis secondary to lovebirds: a new cause of bird fancier's
} disease

\author{
M. Funke* and J-M. Fellrath ${ }^{\#}$
}

ABSTRACT: Hypersensitivity pneumonitis (HP) is an immunologically mediated lung disease due to the repetitive inhalation of antigens. Most new cases arise from residential exposures, notably to birds, and are thus more difficult to recognise.

The present authors report a 59-yr-old male who complained of dyspnoea and cough while being treated with amiodarone. Pulmonary function tests revealed restriction and obstruction with low diffusing lung capacity for carbon monoxide and partial pressure of oxygen. A high-resolution computed tomography chest scan and bronchoalveolar lavage showed diffuse bilateral groundglass attenuation and lymphocytic alveolitis, respectively.

Initial diagnosis was amiodarone pulmonary toxicity, but because of a rapidly favourable evolution, this diagnosis was questioned. A careful environmental history revealed a close contact with lovebirds shortly before the onset of symptoms. Precipitins were strongly positive against lovebird droppings, but were negative against other avian antigens. The patient was diagnosed with hypersensitivity pneumonitis to lovebirds. Avoidance of lovebirds and steroid treatment led to rapid improvement.

The present observation identifies a new causative agent for hypersensitivity pneumonitis and highlights the importance of a thorough environmental history and of searching for precipitins against antigens directly extracted from the patient's environment. These two procedures should allow a more precise classification of some cases of pneumonitis, and thus might avoid progression of active undiagnosed hypersensitivity pneumonitis to irreversible fibrosis or emphysema.

KEYWORDS: Bird fancier's disease, droppings, environmental history, hypersensitivity pneumonitis, lovebirds, precipitins

ypersensitivity pneumonitis (HP) is an immunologically mediated lung disease caused by inhaled antigens that provoke lymphocytic inflammation and granulomatous lesions in the peripheral airways and surrounding interstitium. The only truly effective treatment is early recognition of the causative antigen and control of exposure. Active untreated HP may lead to irreversible fibrosis and/or emphysema.

Many causative agents have been recognised amongst occupational dusts or mists [1], but most current new cases arise from domestic exposures, notably to birds, that are more difficult to recognise. The present article documents the first case of bird fancier's disease caused by lovebirds.

\section{CASE REPORT}

A 59-yr-old Caucasian male was hospitalised because of progressive dyspnoea on exertion, weight loss and a febrile productive cough that was nonresponsive to antibiotics. He had a past history of mild restrictive syndrome secondary to post-operative pleural effusion after mitral valve replacement. The subject was an ex-smoker (90 pack-yrs), and was suffering from atrial fibrillation treated with amiodarone. No pets, including birds, were kept in his house. On admission, physical examination revealed fever and bibasilar inspiratory crackles. Chest radiography showed diffuse bilateral nodular shadowing (fig. 1). A high-resolution computed tomography chest scan showed diffuse bilateral ground-glass opacities
AFFILIATIONS

${ }^{*}$ Clinic and Policlinic of Pulmonary Medicine, University Hospital, Bern, and

\# Service of Respiratory Medicine, University Hospital, Lausanne, Switzerland.

CORRESPONDENCE

J-M. Fellrath

Service of Respiratory Medicine

Centre Hospitalier Universitaire

Vaudois

Rue du Bugnon 46

1011 Lausanne

Switzerland

Fax: 41213140593

E-mail: jean-marc.fellrath@chuv.ch

Received:

August 192007

Accepted after revision:

January 162008

STATEMENT OF INTEREST

None declared. 


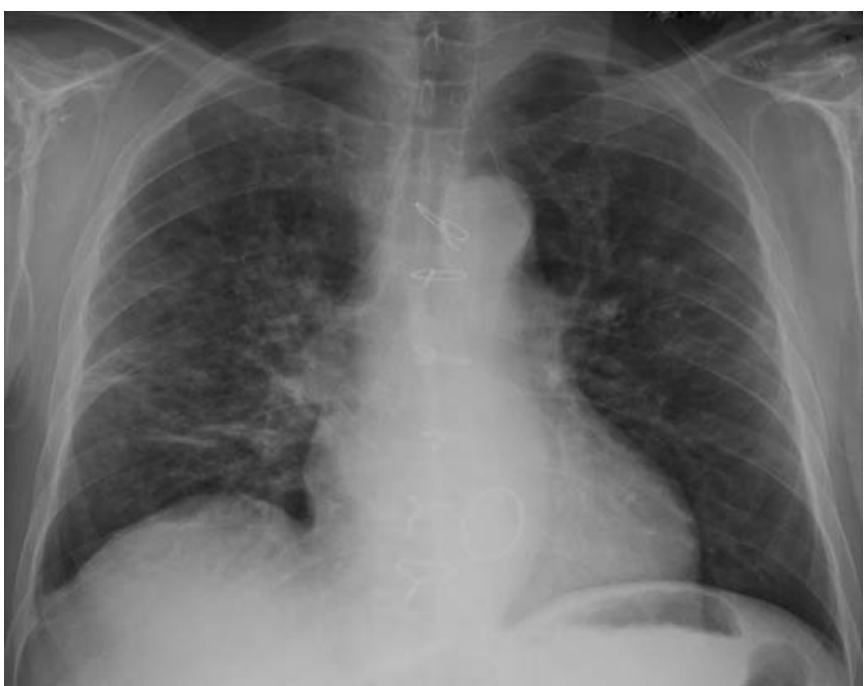

FIGURE 1. Chest radiograph showing diffuse bilateral nodular shadowing.

and fine nodules (fig. 2). The white blood cell count and Creactive protein were $11,800 \mathrm{G} \cdot \mathrm{L}^{-1}$ with $83 \%$ neutrophils and $91 \mathrm{mg} \cdot \mathrm{L}^{-1}$, respectively. Immunological investigations, including rheumatoid factor, antinuclear antibodies and antineutrophil cytoplasmic antibodies were negative. All microbiological investigations remained negative. Pulmonary function tests (PFTs) and arterial blood gas data are summarised in table 1, and were characterised by a mixed pattern of restriction and obstruction with low diffusing capacity of the lung for carbon monoxide $(\mathrm{DL}, \mathrm{CO})$ and partial pressure of oxygen. Flexible bronchoscopy was performed. The bronchoalveolar lavage fluid (BALF) cell count revealed alveolar macrophages of $60 \%$, lymphocytes of $34 \%$, neutrophils of $5 \%$ and eosinophils of $1 \%$, with a low $\mathrm{CD} 4 / \mathrm{CD} 8$ ratio; no foamy macrophages were observed.

A diagnosis of amiodarone pulmonary toxicity (APT) was made and the patient was treated with steroids. However, the

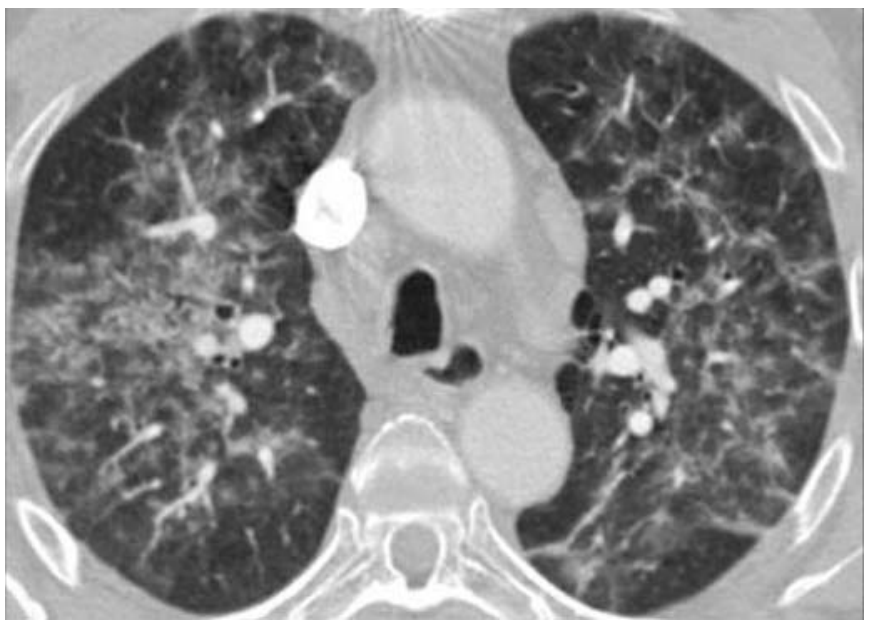

FIGURE 2. High-resolution computed tomography showing diffuse bilateral ground-glass opacities and fine nodules.

\begin{tabular}{|c|c|c|c|c|}
\hline \multirow{3}{*}{\multicolumn{2}{|c|}{$\begin{array}{l}\text { TABLE } 1 \\
\text { Variable }\end{array}$}} & sulmonary fur & ion test and & rterial blood \\
\hline & & \multicolumn{3}{|c|}{ Value } \\
\hline & & On admission & After 2 weeks & After 7 months \\
\hline \multicolumn{2}{|c|}{ FVC L (\% pred) } & $2.03(55)$ & $2.53(69)$ & $2.68(74)$ \\
\hline \multicolumn{2}{|c|}{ FEV1 L (\% pred) } & $1.56(53)$ & $2(70)$ & $1.95(67)$ \\
\hline \multicolumn{2}{|c|}{ FEV $_{1 / \text { FVC } \% \text { (\% pred) }}$} & $77(100)$ & $79(103)$ & $73(95)$ \\
\hline \multicolumn{2}{|c|}{ TLC L (\% pred) } & $4.25(69)$ & $4.62(75)$ & $4.87(80)$ \\
\hline \multicolumn{2}{|c|}{ RV/TLC \% (\% pred) } & $51(138)$ & $44(119)$ & $42(114)$ \\
\hline \multicolumn{2}{|c|}{ DL,COadj (\% pred) } & 39 & 63 & 81 \\
\hline \multicolumn{2}{|c|}{ KcOadj (\% pred) } & 82 & 99 & 111 \\
\hline \multicolumn{2}{|c|}{$\mathrm{PO}_{2} \mathrm{mmHg}^{\# \text {, }}$} & 58 & 76 & 81 \\
\hline \multicolumn{2}{|l|}{$\mathrm{PCO}_{2} \mathrm{mmHg}$} & 36 & 36 & 36 \\
\hline \multicolumn{2}{|c|}{${\mathrm{PA}-\mathrm{a}, \mathrm{O}_{2}}_{\mathrm{mmHg}^{\#,+}}$} & 39 & 24 & 18 \\
\hline \multicolumn{2}{|l|}{$\mathrm{Sa}, \mathrm{O}_{2} \%^{\#}$} & 90 & 94 & 96 \\
\hline
\end{tabular}

FVC: forced vital capacity; \% pred: \% predicted; FEV1: forced expiratory volume in one second; TLC: total lung capacity; RV: residual volume; $D L, C O a d j:$ diffusing capacity of the lung for carbon monoxide adjusted for haemoglobin; KcOadj: transfer coefficient of the lung for carbon monoxide adjusted for haemoglobin; $\mathrm{PO}_{2}$ : partial pressure of oxygen; $\mathrm{PCO}_{2}$ : partial pressure of carbon dioxide; $P$ A$\mathrm{a}, \mathrm{O}_{2}$ : alveolar-arterial oxygen tension difference; $\mathrm{Sa}_{2} \mathrm{O}_{2}$ : arterial oxygen saturation. ${ }^{\#}$ : measured while the patient was breathing room air at $540 \mathrm{~m}$ above sea

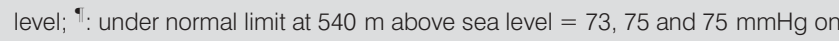
admission, after 2 weeks and after 7 months, respectively; ${ }^{+}$: upper normal limit $22 \mathrm{mmHg}$

current authors questioned the diagnosis of APT due to the following observations: 1 ) the rapid resolution of the fever shortly after the admission, but before the introduction of steroids; 2) the rapid positive response of the respiratory symptoms to steroids; and 3) the absence of foamy macrophages in the BALF. A careful environmental history revealed that the patient had very close daily contact with his daughter's lovebirds shortly before the onset of symptoms. Therefore, precipitin reactions to budgerigar, parrot (macaw) and canary serum using immunoelectrophoresis (Paragon IEP kit; Beckman Coulter, Fullerton, CA, USA) were first performed locally. Due to a negative result, precipitin analysis against a panel of commercial avian antigens (FSK Avian Allergens; Microgen Bioproducts, Camberley, UK) was subsequently performed by a second laboratory, which is expert in serological HP tests (Laboratory of Mycology and Parasitology, University Hospital of Besançon, Besançon, France). This panel included pigeon and budgerigar serum and faecal extract, as well as poultry serum. As this commercial test was also negative, five antigens were produced from lovebird droppings by the laboratory at Besançon for serological testing, including one crude antigen (technique of Pepys [2]) and four soluble antigens purified from microorganisms (Fusarium solani, Absidia corymbifera, Eurotium amstelodami, Eurotium umbrosum) isolated from the droppings culture (modified technique of Symoens [2]). Double immunodiffusion test according to Ouchterlony was performed as a screening test [2]. Sodium tricitrate (5\%) pre-treatment of the gel was 


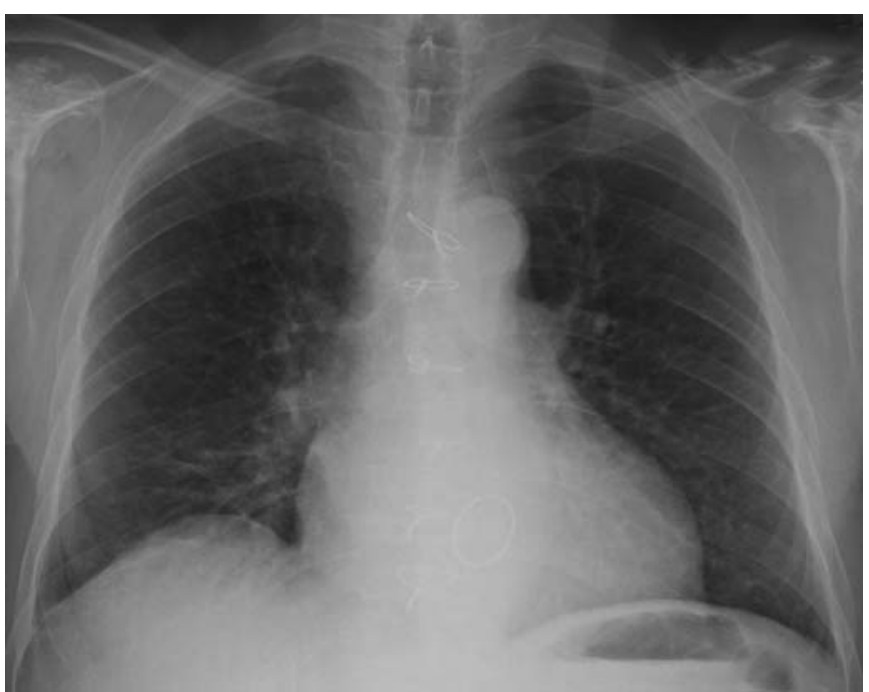

FIGURE 3. Chest radiograph after 7 months of avoidance of contact with lovebirds appears normal.

performed for minimising false-positive results. Electrosyneresis and immunoelectrophoresis were used as confirmation tests [2]. The double diffusion test was positive against pigeon extract, but this result could not be confirmed by immunoelectrophoresis. There was a strong precipitin reaction to lovebird droppings using the double immunodiffusion test (three lines), which was confirmed by electrosyneresis (one line) and immunoelectrophoresis (one line). Among the soluble antigens, only precipitin reaction to A. corymbifera was weakly positive and partially confirmed.

According to these data and the published diagnostic criteria, the patient was thus diagnosed with an acute form of bird fancier's disease. Steroids were maintained on high doses for 2 weeks and than tapered over 6 weeks, and the lovebirds were definitively removed from the patient's environment. After 7 months of complete avoidance from the lovebirds, the patient remained asymptomatic, his chest radiograph was normal (fig. 3) and his PFTs showed both normal DL,CO and arterial blood gases with lung volumes at a lower normal limit (table 1), which was consistent with past history of postoperative restriction.

\section{DISCUSSION}

The bird fancier's disease is probably one of the most prevalent types of HP in western urban areas, even if reliable epidemiological data are not available. Avian pneumonitis is mainly described amongst budgerigar and pigeon fanciers because of their prevalence [3-5]. Feather duvets are also well-known agents $[6,7]$. Anecdotal case reports described cockatiel [8], pheasants [9], canaries [10], parakeets [11], rosella parrots [12], parrots [13], geese [14] and owl exposure [15] associated with HP. The only case report of HP with lovebird exposure presented in a female exposed to a total of eight birds (parrots, parakeets, one cockatoo, one conure and one lovebird), all of them provoked positive precipitins. It is unclear whether the lovebird was partially responsible for the disease in this case [16]. To the current authors' knowledge, there is no previous description of bird fancier's disease exclusively related to lovebird exposure, and they therefore report this new aetiology herein.

Lovebirds belong to the Psittacidae family. This family includes roughly 370 species of birds, to which the general name "parrot" is applied [17]. The level of antigenic crossreactivity is thought to be quite high among these different birds [8]. According to the geographical distribution of these birds, it is accepted from an ornithological point of view to subdivide the Psittacidae family into the following three groups: 1) lovebirds (genus Agapornis) in Africa; 2) budgerigars (genus Melopsittacus) in Oceania; and 3) macaws (genus Ara) in South America. This geographical distribution has certainly had some influence on the evolutionary history of these birds and, as a consequence, on their current phylogenetic distance and serological differences [18]. The current patient showed positive precipitin reaction to lovebird dropping extract, but no precipitin reaction to budgerigar and parrot (macaw) serum, which is thus suggestive of a lovebirdspecific antigen not shared by other species of the Psittacidae family. There was also no precipitin reaction to canary and pigeon serum. The current authors tried to identify the specific source of lovebird antigen more precisely. The present patient showed only a weakly positive precipitin and probably insignificant reaction to one out of four soluble antigens purified from microorganisms isolated from a culture of lovebird droppings. The difference between the results obtained with crude antigen as opposed to soluble antigens could be explained by the difference in the extraction mode and by the presence in droppings of antigens that are not suitable for culture. Finally, unspecific lines were not observed on the serological tests, which ruled out a false-positive result [19]. Taken together, all these data strongly supported the identification of a truly novel antigen as the cause of avian $\mathrm{HP}$, even if there was failure to characterise its specific nature.

The natural evolution of HP is characterised by a chronic and irreversible disease if antigen exposure persists [20]. Bird fancier's disease probably progresses more often to more severe chronic forms than other forms of $\mathrm{HP}$, such as farmer's lung, which can even improve spontaneously [21,22]. The only truly effective intervention that avoids a chronic evolution is early recognition of the causative antigen and control of exposure. Identification of the causative antigen, which is probably the most relevant step in HP diagnosis [22], is certainly challenging and often frustrating, notably in cases of chronic disease where antigenic load can be very low [23] Recognition of the causative antigen relies on a careful environmental history, which should sometimes be reassessed, notably in cases where the following applies: 1) environmental history is not immediately conclusive; 2) the aetiology of a chronic interstitial lung disease (ILD) remains unclear at the term of investigations; and 3) some data, including investigations, clinical course and response to treatment, are discordant for clarifying the aetiology of an ILD.

The present case highlights the importance of a careful recording of environmental history. The patient was indeed known for amiodarone treatment, which naturally initially suggested the diagnosis of amiodarone pulmonary toxicity, as autoimmune data and environmental history were negative. Notably, he denied pets at home (on first questioning, the 
patient was asked, "Do you own pets?"). The rapid improvement immediately after admission and under steroids, and the absence of foamy cells in the BALF were significant [24]. Only a repeated history and modified questioning revealed his recent exposure to his daughter's lovebirds, while his usual environment was pet-free (on second questioning, the patient was asked, "Are you in contact with pets?"). This interrogative detail is essential to establish the diagnosis, as exposure to an offending antigen predicts HP with a very high odds ratio [22]. Furthermore, identification of the possible offending antigen allows a search for specific precipitins using extracts from the patient's environment [22], which is probably more efficient than searching for precipitins against commercial antigens [16]. This dual procedure is very important in the diagnostic process of HP [22]. In the present case, it allowed the diagnosis of APT to be corrected. Without this dual procedure, the present patient's exposure to his daughter's lovebirds would have continued, and thus also his high risk of developing a chronic disease, including respiratory and right heart failure, and even premature death [25], as well as a probable requirement for long-term steroids and immunosuppressive therapy.

In conclusion, the current authors' observation identifies a new causative agent of bird fancier's disease. New hypersensitivity pneumonitis agent determination is useful to help clinicians to identify possible causes of illness and, consecutively, treatment [26]. In addition, it highlights the importance of a carefully taken environmental history and of searching for precipitins against antigens directly extracted from the patient's environment. Only this dual procedure will allow classification of some cases of pneumonitis, either misdiagnosed, as in the present case, or of unknown origin. This in turn could prevent active undiagnosed, and thus untreated, hypersensitivity pneumonitis developing into irreversible fibrosis and/or emphysema.

\section{ACKNOWLEDGEMENTS}

Specific serum precipitin analysis was performed by G. Reboux (Laboratoire de Parasitologie-Mycologie, Centre Hospitalier Universitaire J. Minjoz, Besançon, France).

The authors would like to thank N. Knowlton Mean and T. McKee (Dept of Pathology, University Hospital Geneva, Geneva, Switzerland) for their careful linguistic revision of the manuscript.

\section{REFERENCES}

1 Reboux G, Piarroux R, Mauny F, et al. Role of molds in farmer's lung disease in Eastern France. Am J Respir Crit Care Med 2001; 163: 1534-1539.

2 Reboux G, Magy N, Dalphin JC. Immunological methods. In: Genevois PA, De Vuyst P, eds. Imaging of Occupational and Environmental Disorders of the Chest. 1st Edn. Berlin, Heidelberg, New York, Springer, 2006; pp. 133-158.

3 Hargreave FE, Pepys J, Longbottom JL, Wraith DG. Bird breeder's (fancier's) lung. Lancet 1966; 1: 445-449.

4 Hendrick DJ, Faux JA, Marshall R. Budgerigar-fancier's lung: the commonest variety of allergic alveolitis in Britain. Br Med J 1978; 2: 81-84.
5 Reed CE, Barbee RA. Pigeon-breeders' lung: a newly observed interstitial pulmonary disease. JAMA 1965; 193: 261-265.

6 Inase N, Ohtani Y, Endo J, Miyake S, Yoshizawa Y. Feather duvet lung. Med Sci Monit 2003; 9: CS37-CS40.

7 Inase N, Ohtani Y, Sumi Y, et al. A clinical study of hypersensitivity pneumonitis presumably caused by feather duvets. Ann Allergy Asthma Immunol 2006; 96: 98-104.

8 McCluskey JD, Haight RR, Brooks SM. Cockatiel-induced hypersensitivity pneumonitis. Environ Health Perspect 2002; 110: 735-738.

9 Partridge SJ, Pepperell JC, Forrester-Wood C, Ibrahim NB, Raynal A, Swinburn CR. Pheasant rearer's lung. Occup Med (Lond) 2004; 54: 500-503.

10 Sutton PP, Pearson A, du Bois RM. Canary fancier's lung. Clin Allergy 1984; 14: 429-431.

11 Sahn SA, Richerson HB. Extremes of clinical presentation in parakeet-fancier's lung. Arch Intern Med 1972; 130: 913-917.

12 Caruana M, Cornish KS, Bajada S, Jones CF, Cacciottolo J. Rosella parrot exposure as a cause of bird fancier's lung. Arch Environ Occup Health 2005; 60: 187-192.

13 Liu YN, Chen LA, Zhang ZY, Li QS. Parrot breeder's lung: first case report in China. Chin Med J (Engl) 1989; 102: 947-950.

14 Saltoun CA, Harris KE, Mathisen TL, Patterson R. Hypersensitivity pneumonitis resulting from community exposure to Canada goose droppings: when an external environmental antigen becomes an indoor environmental antigen. Ann Allergy Asthma Immunol 2000; 84: 84-86.

15 Choy AC, Patterson R, Ray AH, Roberts M. Hypersensitivity pneumonitis in a raptor handler and a wild bird fancier. Ann Allergy Asthma Immunol 1995; 74: 437-441.

16 Krasnick J, Meuwissen HJ, Nakao MA, Yeldandi A, Patterson R. Hypersensitivity pneumonitis: problems in diagnosis. J Allergy Clin Immunol 1996; 97: 1027-1030.

17 Collar NJ. Family Psittacidae (Parrots). In: Del Hoyo J, Elliott A, Sargatal J, eds. Handbook of the Birds of the World. Vol. 4, Sandgrouse to Cuckoos. Barcelona, Lynx, 1997; pp. 280-284.

18 Sibley CG, Ahlquist JE. Parrots In: Sibley CG, Ahlquist JE, eds. Phylogeny and Classification of Birds: A Study in Molecular Evolution. New Haven, London, Yale University Press, 1990; pp 380-390.

19 Faux JA, Holford-Strevens V, Wells ID, Pepys J. "False positive" precipitation reactions to extracts of organic dusts due to a teichoic acid from S. aureus. Clin Exp Immunol 1970; 7: 897-902.

20 Perez-Padilla R, Gaxiola M, Salas J, Mejia M, Ramos C, Selman M. Bronchiolitis in chronic pigeon breeder's disease. Morphologic evidence of a spectrum of small airway lesions in hypersensitivity pneumonitis induced by avian antigens. Chest 1996; 110: 371-377.

21 Perez-Padilla R, Salas J, Chapela R, et al. Mortality in Mexican patients with chronic pigeon breeder's lung compared with those with usual interstitial pneumonia. Am Rev Respir Dis 1993; 148: 49-53.

22 Lacasse Y, Selman M, Costabel U, et al. Clinical diagnosis of hypersensitivity pneumonitis. Am J Respir Crit Care Med 2003; 168: 952-958. 
23 Navarro C, Mejia M, Gaxiola M, Mendoza F, Carrillo G, Selman M. Hypersensitivity pneumonitis: a broader perspective. Treat Respir Med 2006; 5: 167-179.

24 Bedrossian CW, Warren CJ, Ohar J, Bhan R. Amiodarone pulmonary toxicity: cytopathology, ultrastructure, and immunocytochemistry. Ann Diagn Pathol 1997; 1: 47-56.
25 Solaymani-Dodaran M, West J, Smith C, Hubbard R. Extrinsic allergic alveolitis: incidence and mortality in the general population. QJM 2007; 100: 233-237.

26 Girard M, Israel-Assayag E, Cormier Y. Pathogenesis of hypersensitivity pneumonitis. Curr Opin Allergy Clin Immunol 2004; 4: 93-98. 